\title{
Mixed top-bottom squark production at the LHC
}

\author{
D. Berdine $\mathrm{B}^{1, *}$ and D. Rainwater ${ }^{1, \dagger}$ \\ ${ }^{1}$ Dept. of Physics and Astronomy, \\ University of Rochester, Rochester, NY 14627
}

(Dated: January 2, 2019)

\begin{abstract}
We calculate cross sections for mixed stop-sbottom pair production at the LHC, analogous to single-top production, a weak process involving the $W-\tilde{t}_{i}-\tilde{b}_{j}$ vertex. While coupling-suppressed relative to QCD same-flavor squark pair production, the signal is distinctive due to heavy-flavor tagging along with a possible same-sign lepton pair in the final state. SUSY backgrounds can often be suppressed many orders of magnitude by taking advantage of distinct kinematic differences from the signal. Measuring the rate of this process would add significant additional information to that gathered from other SUSY processes. If the stop and sbottom mixings can be determined elsewhere, stop-sbottom production would provide for a measurement of the weak squark gauge coupling and super-CKM vertex factor.
\end{abstract}

*Electronic address: berdine@pas.rochester.edu

${ }^{\dagger}$ Electronic address: rain@pas.rochester.edu 


\section{INTRODUCTION}

Spacetime Supersymmetry (SUSY) has become one of the leading candidate extensions to the Standard Model (SM) of particle physics [1]. Besides the aesthetic appeal of maximally extending the Poincaré group [2], the Minimal Supersymmetric Standard Model (MSSM) also provides a natural weakly-interacting dark matter candidate, a solution to the Higgs sector naturalness problem, and likely unification of gauge couplings near the Planck scale, among other features.

If SUSY exists, it must be a broken symmetry at low energy, as we do not observe the opposite-spin-statistics partners of the SM field content which would necessarily exist. As a result, the squarks, sleptons, charginos and neutralinos of the MSSM must be fairly massive in comparison to their SM counterparts. Previous high-energy physics experiments such as LEP and Run I of the Tevatron have put stringent bounds on some of the sparticle masses. It will fall to the CERN LHC proton-proton collider to perform a conclusive SUSY search, although the real physics would only begin were a potential SUSY discovery to be made.

If LHC finds candidate SUSY particles, a great deal of work would be required to prove or disprove the hypothesis that the new particle content belongs to the SUSY partner spectrum of the SM content, and if confirmed to perform a sufficient number of measurements to determine the parameters of the SUSY Lagrangian 3, 4, 5]. For the LHC the prospects of undertaking both tasks is uncertain, since many of the weakly-interacting spartners might go unobserved, and many of the colored spartners would have overlapping signatures; disentangling these would be a daunting prospect. The LHC will also be hard pressed to measure sparticle masses: for some, only mass differences may be accessible [5, 6, 7, 8]. Performing such tasks as measuring the spins of new state, or confirming that their couplings are indeed identical to the gauge and Yukawa couplings of the SM, is largely unexplored territory phenomenologically. Ideally, a future linear collider (ILC) will be constructed which could address many of these precision measurement problems [9], and there is considerable effort to understand the synergy that would exist between LHC and ILC [10]. However, there is also now the planned luminosity upgrade to LHC, the SLHC [11]. While it certainly won't be able to bring about a precision-era of physics and guarantee disentanglement of whatever new physics is found at LHC, our goal here is to explore just how far LHC and SLHC could push the envelope in measurements of the new physics.

SUSY phenomenology at LHC has so far focused mainly on the dominant $2 \rightarrow 2$ processes, which for colored sparticles is QCD production of gluino pairs, same-flavor squark pairs, and mixed gluino-squark pairs. These production channels probe only the SUSY-QCD vertices. While the sparticle cascade decays will necessarily involve weak-interaction vertices, the actual measurement is the number of events resulting from the production cross section times branching ratio (BR) to a given final state. This alone is not enough information to separate the different vertices. ${ }^{1}$ Analogous to the case of single-top production in the SM 12], observing mixed-flavor squark production, which occurs via the weak vertex $W-\tilde{q}_{L^{-}}$ $\tilde{q}_{L}^{\prime}$ and semi-weak vertex $W-g-\tilde{q}_{L^{-}} \tilde{q}_{L}^{\prime}$, would add additional information about the sparticle interaction vertices. Note that the weak interaction, being left-handed, involves only the lefthanded squark partners of the quarks. In fact, assuming that the various sparticle BRs were determined in QCD pair production, mixed-flavor production would then provide absolute

\footnotetext{
${ }^{1}$ In principle, the QCD vertex could be measured by observing all possible sparticle decays, so that the total rate observed is $100 \%$ of the $\mathrm{BR}$.
} 
measurements of the weak vertex couplings.

Weak production processes are naïvely approximately two orders of magnitude smaller than QCD production processes - although this also depends on the relative mass spectrum with respect to which parton luminosity dominates for that regime of $x$. This would present a problem for identifying this mixed-flavor signal. There is some hope, however. In maximally-unifying scenarios, a.k.a. "GUT-inspired", over much of parameter space the colorless sparticles would be lighter than the colored sparticles. The cascade decay chains of squarks would typically therefore proceed ultimately through the lightest slepton (almost always a stau in popular unifying scenarios) or lightest chargino, in either case giving a final-state lepton ${ }^{2}$. Mixed-flavor production often preferentially gives same-sign leptons in the final state, compared to opposite-sign leptons from QCD pair production. Since the ATLAS 13 and CMS 14 detectors at LHC will be able to identify lepton charge sign to better than $10^{-4}$ accuracy, this characteristic of weak mixed-flavor production could provide a separation from the much greater rate of QCD production events. Our physics goal is to find and exploit characteristics of mixed-flavor squark production which would make these weak cross sections stand out against the much larger QCD squark production processes, providing a way to measure the weak vertices.

\section{STOP-SBOTTOM PRODUCTION AT A HADRON COLLIDER}

The best hope to look for mixed-flavor squark production is in the third generation, i.e. top and bottom squarks. The reasons for this are threefold. First, the largest first- and second-generation squark pair production mechanism at LHC is typically $u u \rightarrow \tilde{u}_{L} \tilde{u}_{L}$, which proceeds via a $t$-channel Majorana gluino and dominates over squark-antisquark production because of the very high initial-state valence $u$ quark luminosity. This state would decay predominately to same-sign leptons via $\tilde{u}_{L} \rightarrow \chi_{1}^{+} d$, with the pair of lightest charginos, $\chi_{1}^{+}$, decaying to same-sign leptons. Second, the first- and second-generation squarks are impossible to cleanly separate from each other and from gluino pair and gluino-squark production, due to the inability to tag light flavors. Tagging the $b$ jets in stop-sbottom mixed pairs would eliminate the first- and second-generation squark contributions, up to the level of fake $b$ tag rates. Third, because gluinos are Majorana, their decay chains give equal probability for either fermion sign [15]. As a result, gluino pairs and squark-gluino production would be large sources of same-sign leptons. Tagging $b$ jets can likewise reduce this fake source of same-sign leptons, except that, depending on the spectrum, gluinos may decay into stops or sbottoms themselves. We will come back to these issues later as background considerations.

For the third generation, the left- and right-handed squarks $\tilde{q}_{L}, \tilde{q}_{R}$ can mix, forming mass eigenstates $\tilde{q}_{1}, \tilde{q}_{2}$, with $\tilde{q}_{1}$ defined as lighter. The mixing is proportional to the Yukawa couplings of the SM fermions. The general mass matrix for stops is given by:

$$
M_{\tilde{t}}^{2}=\left(\begin{array}{cc}
M_{\tilde{t}_{L}}^{2} & +m_{t}\left(A_{t}^{*}-\mu \cot \beta\right) \\
+m_{t}\left(A_{t}-\mu^{*} \cot \beta\right) & M_{\tilde{t}_{R}}^{2}
\end{array}\right)
$$

where $\tan \beta$ is the ratio of Higgs sector vacuum expectation values $v_{u} / v_{d}, \mu$ is the Higgsino

\footnotetext{
${ }^{2}$ A third possibility exists, where the cascade proceeds ultimately through an on-shell $Z$ boson plus LSP.

This scenario in general presents problems for SUSY analyses in disentangling production processes.
} 
mass parameter, $A_{t}$ is the top squark trilinear scalar coupling in the soft SUSY breaking potential, and

$$
\begin{aligned}
& M_{\tilde{t}_{L}}^{2}=m_{\tilde{t}_{L}}^{2}+M_{Z}^{2} \cos 2 \beta\left(I_{3 t}-s_{W}^{2} Q_{t}\right)+m_{t}^{2}, \\
& M_{\tilde{t}_{R}}^{2}=m_{\tilde{t}_{R}}^{2}+M_{Z}^{2} \cos 2 \beta s_{W}^{2} Q_{t}+m_{t}^{2} .
\end{aligned}
$$

where $m_{\tilde{q}_{L}}, m_{\tilde{q}_{R}}$ are the soft-breaking masses in the SUSY Lagrangian. For $b$ instead of $t$, replace $\cot \beta$ with $\tan \beta$. Because of this $L-R$ mixing in the third generation, the SUSY weak vertices $W-\tilde{t}_{i}-\tilde{b}_{j}$ are more complicated than the corresponding $W-t-b$ vertex in the SM: they contain not just the super-CKM angle $\widetilde{V}_{t b}$, but also the mixing angles of the stops and sbottoms, which reflect the left-handed component of each squark. Expressed in terms of reduced parameters, the $W-\tilde{t}_{1}-\tilde{b}_{1}$ coupling is $g_{W} \widetilde{V}_{t b} \cos \theta_{t} \cos \theta_{b}$, where the mixing angles are those that diagonalize Eq. 1 for stop and sbottom squarks, respectively. For $\tilde{q}_{2}$ instead of $\tilde{q}_{1}, \cos \theta$ is replaced by $-\sin \theta$ to obtain the left-handed component. If SUSY were an exact symmetry, $\widetilde{V}_{t b} \equiv V_{t b}$. This is a very good approximation even after SUSY breaking and evolution down to the electroweak scale [16, 17], although it can be altered if SUSY breaking is not flavor-blind.

As in SM single-top production, there are three ways to produce mixed-flavor squarks at LHC: $s$-channel, $t$-channel, and $W$-associated production. The first two are shown in Figs. [1 and 2, respectively. The third is typically about $1 / 3$ of the $t$-channel rate, but would be likely be experimentally indistinct from QCD sbottom pair production. We will therefore ignore this channel. The analogy to SM single-top production is not complete, since in the SM case the light $b$ quark mass allows it to be treated as an initial-state parton, which cannot happen here. The SM process also involves only one heavy state, whereas here we have two, leading to different kinematics. Finally, in the SUSY case, because the squarks are scalars, there is a four-point vertex which does not exist in the SM. Although the graphs of Fig. 2 are part of the real-emission QCD corrections to the basic process of Fig. 1 they behave very differently. As we will see, the $t$-channel cross sections are always much larger than the $s$-channel. This is a combination of the larger suppression from an $s$-channel propagator at large squark pair invariant mass, and initial-state gluon versus sea quark luminosity.

To calculate cross sections, we use matrix elements generated by a new MSSM version of MADGRAPH [18], called SMADGRAPH [19]. Our calculations utilize CTEQ6L1 structure functions [20] for the incoming protons at LHC, $\sqrt{s}=14 \mathrm{TeV}$, and we choose the average of the two final-state squark masses as the factorization and renormalization scales. We begin by analyzing the cross sections for the MSSM parameter space benchmark point ${ }^{3}$ SPS1a [21]. For this point, the squark masses are $m_{\tilde{t}_{1}, \tilde{t}_{2}}=396,587 \mathrm{GeV}$ and $m_{\tilde{b}_{1}, \tilde{b}_{2}}=517,547 \mathrm{GeV}$.

As seen in Table In the LO total cross sections for SPS1a are typically quite small, on the order of a few fb, although even this would produce many hundreds of events for the planned luminosity of $300 \mathrm{fb}^{-1}$ per experiment at the LHC, or thousands of events for the $3000 \mathrm{fb}^{-1}$ per experiment for the planned luminosity upgrade to the LHC, the SLHC [1]

(this would be, after all, a long-term measurement to determine the SUSY Lagrangian parameters, not a discovery channel). Note the asymmetry in $\tilde{t}^{*}$ v. $\tilde{t}^{*} \tilde{b}$ production. It

\footnotetext{
${ }^{3}$ We use the SPS benchmarks 21], designed to represent a number of canonical scenarios to aid exploratory phenomenology, only as examples for convenience, not as any suggestion that these scenarios are more likely to be realized in nature than any other. They should be regarded only as starting points for phenomenological investigation.
} 


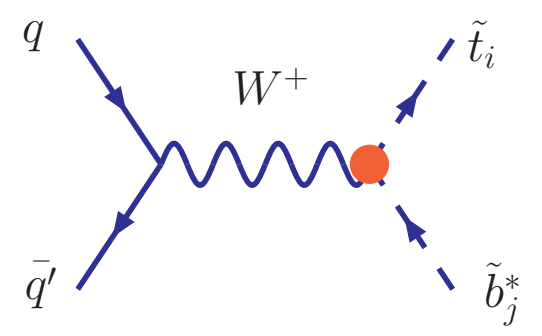

FIG. 1: Feynman diagram for $s$-channel $\tilde{t}_{1} \tilde{b}_{1}^{*}$ production at a hadron collider.
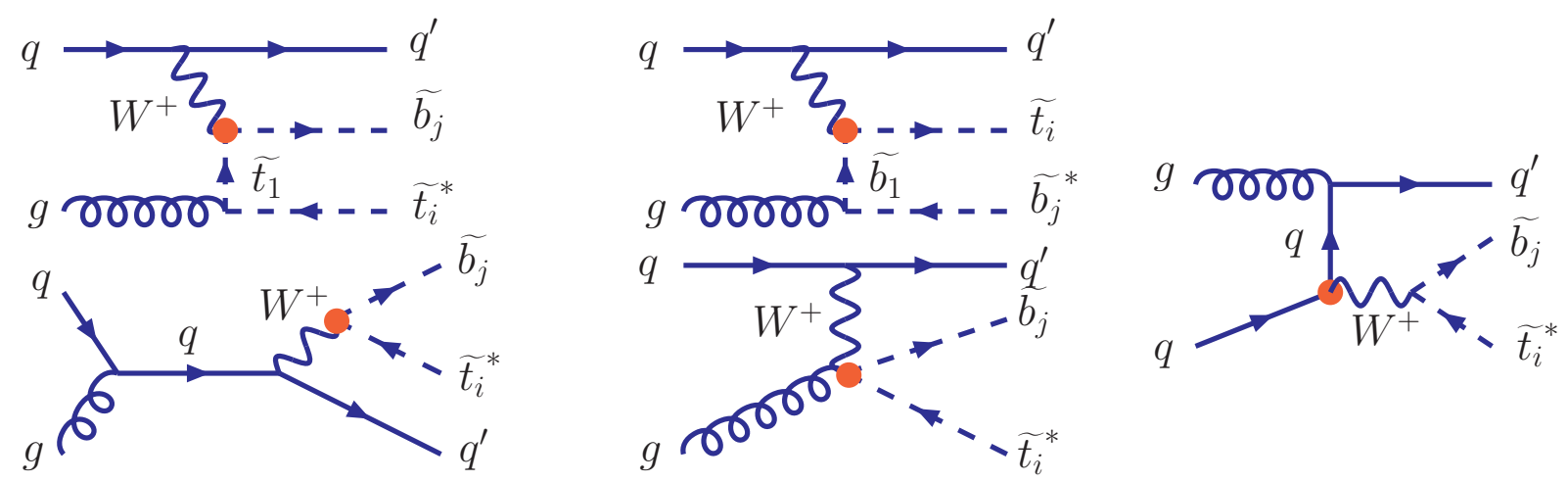

FIG. 2: Feynman diagrams for $t$-channel $\tilde{t}_{i} \tilde{b}_{j}^{*}$ production at a hadron collider. The dot highlights the weak vertex to be measured.

is due to the PDF asymmetry of the incoming protons, LHC being a $p-p$ collider, and the dominance (by approximately a factor of two) of initial-state up quarks over down quarks. Table【also reveals the left-handed and right-handed components of the stops and sbottoms. Since $\tilde{b}_{1}$ and $\tilde{b}_{2}$ are nearly degenerate, the relative ratio of their cross sections shows that it is $\tilde{b}_{1}$ which is mostly $\tilde{b}_{L}$. In contrast, while $m_{\tilde{t}_{2}} \gg m_{\tilde{t}_{1}}$, the $\tilde{t}_{1}$ and $\tilde{t}_{2}$ cross sections are nearly the same, which shows that $\tilde{t}_{2}$ is more left-handed, although not by as much of a margin as in the sbottoms. In SUSY models where the mass parameters are unified at the GUT scale, it is a general feature that the lighter stop is more right-handed and the lighter sbottom is more left-handed. This comes about from the renormalization-group running of the mass parameters from the GUT to the TeV scale due to the different quantum numbers of top and bottom [22].

We give the total $\tilde{t} \tilde{b}$ cross sections in Table $[1]$ combining $s$ - and $t$-channel results and all $\tilde{q}_{1}$ and $\tilde{q}_{2}$ combinations, for all 10 SPS points, along with the stop and sbottom masses at each point. For comparison, we also show the total $\tilde{q}_{1} \tilde{q}_{1}^{*}+\tilde{q}_{2} \tilde{q}_{2}^{*}$ QCD production rates, calculated here with SMADGRAPH, although this was first performed in Ref. [23]. The general feature is

\begin{tabular}{|c|c|c|c|c|}
\hline & $\tilde{t}_{1} \tilde{b}_{1}$ & $\tilde{t}_{1} \tilde{b}_{2}$ & $\tilde{t}_{2} \tilde{b}_{1}$ & $\tilde{t}_{2} \tilde{b}_{2}$ \\
\hline$s$-ch., $\tilde{t} \tilde{b}^{*}$ & 1.3 & .18 & 1.4 & .20 \\
\hline$s$-ch., $\tilde{t}^{*} \tilde{b}$ & .51 & .07 & .51 & .07 \\
\hline$t$-ch., $\tilde{t} \tilde{b}^{*}$ & 4.7 & .73 & 3.6 & .47 \\
\hline$t$-ch., $\tilde{t}^{*} \tilde{b}$ & 2.2 & .34 & 1.6 & .21 \\
\hline
\end{tabular}

TABLE I: Cross sections [fb] for mixed stop-sbottom production at LHC at MSSM benchmark point SPS1a. For the $t$-channel results, we do not impose a kinematic cut on the final-state quark. 


\begin{tabular}{|c||c|c|c|c||c|c|c|c|c|c|c|}
\hline SPS & $m_{\tilde{t}_{1}}$ & $m_{\tilde{t}_{2}}$ & $m_{\tilde{b}_{1}}$ & $m_{\tilde{b}_{2}}$ & $\tilde{t}^{*}$ & $\tilde{t}^{*} \tilde{b}$ & $\tilde{t}^{*}$ & $\tilde{b} \tilde{b}^{*}$ & $\tilde{g} \tilde{g}$ & $\tilde{u}_{L} \tilde{g}$ & $\tilde{d}_{L} \tilde{g}$ \\
\hline $1 \mathrm{a}$ & 396 & 587 & 517 & 547 & 12.6 & 5.49 & 1630 & 560 & 4900 & 7370 & 3740 \\
$1 \mathrm{~b}$ & 653 & 839 & 778 & 828 & 1.25 & 0.468 & 95.9 & 44.7 & 238 & 582 & 260 \\
2 & 943 & 1318 & 1308 & 1567 & 0.045 & 0.014 & 7.74 & 0.74 & 780 & 128 & 52 \\
3 & 641 & 842 & 793 & 824 & 1.26 & 0.474 & 106 & 41.1 & 245 & 623 & 279 \\
4 & 540 & 696 & 619 & 691 & 3.74 & 1.52 & 306 & 168 & 1350 & 1890 & 897 \\
5 & 248 & 648 & 563 & 651 & 26.4 & 12.0 & 14200 & 280 & 1560 & 2780 & 1340 \\
6 & 476 & 704 & 640 & 662 & 3.90 & 1.59 & 575 & 164 & 1570 & 2510 & 1210 \\
7 & 802 & 894 & 861 & 878 & 0.620 & 0.223 & 31.8 & 25.1 & 218 & 527 & 234 \\
8 & 974 & 1091 & 1063 & 1082 & 0.167 & 0.055 & 8.16 & 5.68 & 500 & 400 & 174 \\
9 & 940 & 1152 & 1121 & 1278 & 0.139 & 0.046 & 8.94 & 2.76 & 43 & 85 & 34 \\
\hline
\end{tabular}

TABLE II: Stop and sbottom masses $[\mathrm{GeV}]$, third-generation squark LHC pair production LO cross sections [fb] for both mixed-flavor EW and same-flavor QCD, and gluino pair and squarkgluino production, for the various MSSM benchmark SPS points. We combine $s$ - and $t$-channel cross sections for the signals, without any kinematic cut on the final-state light quark in the $t$-ch. processes. We also combine all stop-sbottom combinations in the total EW rates, and the $\tilde{q}_{1} \tilde{q}_{1}^{*}+\tilde{q}_{2} \tilde{q}_{2}^{*}$ rates for QCD. For squark-gluino production, what we call $\tilde{u}_{L}$ is actually $\tilde{u}_{L}+\tilde{c}_{L}+\tilde{d}_{L}^{*}+\tilde{s}_{L}^{*}$, which together compose the contributions which would give a $\chi^{+}$in their decays; similarly for $\tilde{d}_{L} \tilde{g}$ production.

that rates are lower for heavier squarks, as expected due to phase space suppression, with an additional but non-obvious suppression from mixings. SPS1a has larger cross sections than most other SPS points, except for the light-stop scenario of SPS5. The questions are now, given some rather small rates, might any of these produce enough events to potentially be observable, what are the backgrounds in each potentially viable scenario, and what would measuring these cross sections tell us about SUSY?

Before addressing the signal and background rates, we make a few comments on the significance of a potential stop-sbottom rate measurement. Ideally, one could measure the rate for each stop-sbottom pair independently and with good accuracy. One could then extract each of $g_{W} \tilde{V}_{t b} \cos \theta_{t} \cos \theta_{b}, g_{W} \tilde{V}_{t b} \cos \theta_{t} \sin \theta_{b}, g_{W} \tilde{V}_{t b} \sin \theta_{t} \cos \theta_{b}$ and $g_{W} \tilde{V}_{t b} \sin \theta_{t} \sin \theta_{b}$ independently. The sum of the squares of these is simply $g_{W}^{2} \tilde{V}_{t b}^{2}$, so this combination would in principle be a test of SUSY, as it would measure whether or not the weak $W$ - $\tilde{t}-\tilde{b}$ vertex is of the same strength as the SM weak vertex $W-t-b$. Measurement of the weak coupling strength in only a single channel, or couple of channels, would be muddled by the mixing between left and right states. If this were all that was available, one would then argue that the measurement aids determination of the mixing angles.

However, both of these ideas rely on the GUT-inspired model assumption of flavordiagonal SUSY soft-breaking masses. This is not necessarily strongly motivated, as presumably flavor physics enters at the GUT scale if not before, and could well cause a deviation from such unifying assumptions. If the terms are not diagonal, then the mass matrices which diagonalize all the squarks mix the first two generations with the third, resulting in $\tilde{V}_{t b} \neq V_{t b}$, and the stop-sbottom cross section(s) would help reveal the nature of the SUSY soft-breaking terms. Of course, in this case, all squarks would have some decay branching fraction to heavy flavor, which may be observable. This is obviously a much more complicated scenario, which we ignore in this first analysis for simplicity. 


\section{PROJECTIONS FOR SOME SUSY SCENARIOS}

Here we estimate the observability of stop-sbottom production in various scenarios at LHC or its luminosity upgrade, the SLHC. We begin with the largest cross section of all the SPS points, SPS5, then successively discuss SPS1a and various non-SPS points we formulate, ranging from small variations on the SPS points to arbitrary non-universal inputs scenarios motivated by phenomenology of the low-scale MSSM spectrum [22].

\section{A. SPS5 \\ 1. Signal}

We examine the SPS5 scenario first, as it has the largest stop-sbottom cross section of the SPS points. This is due primarily to the lighter stop being only $250 \mathrm{GeV}$, so the final state is not as phase space restricted as in most of the SPS scenarios, which typically have much more massive stops. In SPS5, we see that $\tilde{t}_{1}$ always decays as $\tilde{t}_{1} \rightarrow b \chi_{1}^{+}$, while the heavier stop decays this way $16 \%$ of the time, $57 \%$ to $Z \tilde{t}_{1} \rightarrow Z b \chi_{1}^{+}$, and $20 \%$ to $h \tilde{t}_{1} \rightarrow h b \chi_{1}^{+}$, while the remaining fraction goes to $t \chi_{1,2}^{0}$. The lighter sbottom decays mostly to $W^{-} \tilde{t}_{1}(77 \%)$, with $13 \%$ to $t \chi_{1}^{-}$and the remaining fraction to $b \chi_{2}^{0}$. The heavier sbottom has only a rare $3 \%$ decay to $t \chi_{1}^{-}$, preferring to go to $b \chi_{1}^{0}$ and $W^{-} \tilde{t}_{1}$ approximately equally. We summarize the relevant sparticle masses, total widths at NLO in QCD, and branching fractions relevant for this analysis in Table III.

The decay combination we envision as being distinctive and indicative of stop-antisbottom production is $\tilde{t} \rightarrow b \chi_{1}^{+}$and $\tilde{b}^{*} \rightarrow \bar{t} \chi_{1}^{+}$, yielding $b \bar{t} \chi_{1}^{+} \chi_{1}^{+}$(and its charge-conjugate for antistopsbottom production). Having a top quark to reconstruct (hadronically) in the final state is, we feel, more distinctive than the $b \bar{b} \chi_{1}^{+} \chi_{1}^{-} W^{ \pm}$state from sbottom decay to stop plus a $W$ boson, although this option could be explored as it would enlarge the signal sample considerably if accessible. We ignore decays to neutralinos, because they are charge-neutral and therefore cannot help distinguish $\tilde{q}$ from $\tilde{q}^{*}$.

For SPS5, and typically in most minimal-supergravity (mSUGRA) scenarios, the lightest chargino decays preferentially to a neutrino plus the lightest slepton, typically the lightest stau, $\tilde{\tau}_{1}^{ \pm}$, as here. The stau in turn decays to the lightest SUSY particle (LSP), typically the lightest neutralino, plus a tau. The detector signature would then be $b \bar{b} j j \tau^{ \pm} \tau^{ \pm}+E_{T}$. Both $b$ jets would be tagged by the vertex detector, with an efficiency of about $50 \%$ each [13, 14]. The taus can then in turn be identified by their leptonic decay $\left(34 \%\right.$ BR with $\epsilon_{\mathrm{ID}}=0.95$ ) or their 1-prong hadronic decay $\left(50 \% \mathrm{BR}\right.$ and $\epsilon_{\mathrm{ID}}=32 \%$ ) 24]. For both cases, the charge can be identified with uncertainty smaller than $10^{-4}$, which is near-perfect, and sufficiently small to not worry about fake same-sign events from opposite-sign backgrounds. For the 1/4 of the time in the SPS5 scenario that each chargino decays instead to LSP plus $W$ boson, the leptonic decay of the latter would simply add to the final state, although it would alter the expected ratio of $e$ or $\mu$ to hadronic $\tau$ which would come from $\tilde{\tau} \rightarrow \tau \chi_{1}^{0}$ decays only. For LHC only, considering $600 \mathrm{fb}^{-1}$ by combining the results of two experiments, and combining stau and tau decay modes, we could expect about 550 signal events in the $(++)$ channel before kinematic cuts, or 5500 at SLHC. This is promising, but must be put in the context of visibility above various backgrounds. 


\begin{tabular}{|c|c|c|c|}
\hline & $m$ & $\Gamma_{\mathrm{NLO}}$ & $\mathrm{BR}\left(q \chi_{1}^{+}\right)$ \\
\hline$\tilde{t}_{1}$ & 248 & 0.0365 & $100 \%$ \\
$\tilde{t}_{2}$ & 648 & 14.4 & $17.3 \%$ \\
$\tilde{b}_{1}^{*}$ & 563 & 14.4 & $14.3 \%$ \\
$\tilde{b}_{2}^{*}$ & 651 & 0.635 & $3.9 \%$ \\
$\tilde{u}_{L}$ & 680 & 6.56 & $66.0 \%$ \\
$\tilde{d}_{L}^{*}$ & 684 & 6.46 & $65.4 \%$ \\
\hline
\end{tabular}

\begin{tabular}{|c|c|c|c|c|}
\hline & $m$ & $\Gamma_{\mathrm{NLO}}$ & $\mathrm{BR}\left(b \tilde{b}_{1}^{*}\right)$ & $\mathrm{BR}\left(\tilde{t} \tilde{t}_{1}\right)$ \\
\hline$\tilde{g}$ & 721 & 10.9 & $9.74 \%$ & $26.6 \%$ \\
\hline \hline & $m$ & $\Gamma_{\mathrm{LO}}$ & $\mathrm{BR}\left(\nu_{\tau} \tilde{\tau}^{+}\right)$ & $\mathrm{BR}\left(W^{+} \chi_{1}^{0}\right)$ \\
\hline$\chi_{1}^{+}$ & 230 & 0.010 & $71.4 \%$ & $28.6 \%$ \\
\hline \hline & $m$ & $\Gamma_{\mathrm{LO}}$ & $\mathrm{BR}\left(\tau \chi_{1}^{0}\right)$ & \\
\hline$\tilde{\tau}_{1}^{+}$ & 184 & 0.297 & $100 \%$ & \\
\hline
\end{tabular}

TABLE III: MSSM particle masses and widths [GeV], and important branching ratios for scenario SPS5. The LSP is the lightest neutralino, $\chi_{1}^{0}$, with mass $120 \mathrm{GeV}$.

\section{Backgrounds}

There are multiple sources of backgrounds giving the same or similar final states. From the SM there is $t \bar{t} W^{+}\left(t \bar{t} W^{-}\right)$production [25], which has a total cross section at leading order (LO) of 290(136) fb at LHC, or 2.47(1.16) fb after decay BRs to $b \bar{b} j j \tau^{+} \tau^{+}+\mathbb{E}_{T}$, but before imposing any kinematic cuts. For a comparison with the case of signal taus decaying hadronically these are the relevant cross sections, and slightly larger than the signal, but within a factor of two. If the taus in the signal are to be observed in the lepton-hadron or dual lepton modes, then the SM background would be a factor 4 larger, although the signal would be a factor 5 larger. After cuts the $t \bar{t} W$ contribution is likely to be much smaller than the signal, as $b$ jets and taus and the reconstructed top quark from stop-sbottom decays will be produced much harder, i.e. with significantly more transverse momentum in the detector. More significantly, the SUSY signal is likely to have significantly more missing transverse momentum. It is probable that one can impose cuts such that most of the signal but very little of the SM background survives. However, this will require future complete decay simulation with kinematic cuts.

QCD sbottom pair production can also give a similar final state: $\tilde{b}_{1} \rightarrow W^{-} \tilde{t}_{1} \rightarrow W^{-} b \chi_{1}^{+}$ and $\tilde{b}_{1}^{*} \rightarrow \bar{t} \chi_{1}^{+}$, yielding $b \bar{t} \chi_{1}^{+} \chi_{1}^{+}+W^{-}$. The cross section for sbottoms pairs to this state of decays is $20.7 \mathrm{fb}$ at LO, compared to $3.0 \mathrm{fb}$ for the signal. This reveals a weakness of our proposal, which is that if in a given scenario the sbottom decays to stop with significant $\mathrm{BR}$, then it could potentially fake the signal. However, this final state contains an extra $W$ boson, which could be vetoed in any of its decay modes. Previous applications of such a veto [26] typically achieved more than an order of magnitude reduction, but this applied to processes such as top quark pair production, where the $W$ is produced with little kinematic boost. Here, the $W$ is the decay product of a $560 \mathrm{GeV}$ object decaying to the $W(80 \mathrm{GeV})$ plus a $250 \mathrm{GeV}$ object: the $W$ is likely to be boosted significantly, increasing the veto probability, although an exact calculation with kinematic cuts on the final-state $W$ decay products would be required to determine the precise rejection efficiency. Note that such a veto would also remove most of the $W$-associated signal channel. Hence, for this analysis we are jusitifed in not considering it. As for QCD stop pair production, while it is large, it arises almost exclusively from $\tilde{t}_{1} \tilde{t}_{1}^{*}$ pairs, which decay $100 \%$ to opposite-sign charginos. We find that $\tilde{t}_{2} \tilde{t}_{2}^{*}$ decays to the signal signature are much less than a $1 \%$ background to the signal rate.

Finally, SUSY QCD production of a first- or second-generation squark plus a gluino, as well as gluino pairs, constitutes a potentially very large background. The reason is that squarks decay with large BR to quark plus chargino, and gluinos in SPS5 are sufficiently 
massive to decay to either top-stop or bottom-sbottom. Because gluinos are Majorana particles, they decay equally to particle-sparticle pairs of either sign permutation. This results in a very large cross section for same-sign lepton pairs from squark+gluino and gluino pair decays [15]. To give an example, the largest squark-gluino cross section is $\tilde{u}_{L} \tilde{g}$, due to the initial state containing a valence $u$ quark ${ }^{4}$. For SPS5 this alone has a cross section of $2300 \mathrm{fb}$ at LO, with NLO QCD corrections being a factor 1.3 [27]. The relevant decays are $\tilde{u}_{L} \rightarrow d \chi_{1}^{+}(66 \%)$ and $\tilde{g} \rightarrow \tilde{t}_{1}, b \tilde{b}_{1}^{*}(27 \%, 10 \%)$. These decays produce identifiable final-state content identical to the $t$-channel signal if the extra jet is observed: $j_{h} b \bar{b} j j \tau^{+} \tau^{+}+\mathbb{E}_{T}$, where $j_{h}$ represents a very hard jet which arises from the $d$ quark. This jet is highly energetic due to the large mass difference between the squark and chargino, $680 \mathrm{GeV}$ v. $230 \mathrm{GeV}$. Gluino pair production is $1600 \mathrm{fb}$ at LO, with NLO QCD corrections of a factor 1.9 for the SPS5 gluino and squark masses [27]. The background arises from one gluino decaying to quark plus squark (first- or second-generation only), while the other decays as in the squarkgluino case. The quark jet from the first gluino's decay to quark plus squark, however, is typically soft for SPS5, so ultimately the signature is essentially the same as in squarkgluino production, but with a factor two for gluino decay combinatorics, and another factor two for decay to either up (down) or charm (strange) quark-squark pairs. We would not propose attempting to distinguish this background from the presence of the extra soft jet, as an additional soft jet is likely to arise also in a significant fraction of the signal simply due to QCD radiation. Thus, the heavy squark+gluino backgrounds are 3.8 and $1.9 \mathrm{pb}$ for the $(++)$ and $(--)$ cases, respectively. These are a factor 240 larger than the signal after squark and gluino decays to chargino plus sbottom. Corrections to this from squark pair production with mistagged $b$ jets will be quite small, as the light quark rejection factor for $b$ jet fakes is $1 / 140$. We do not consider this latter source further here.

\section{Differentiating signal and background}

Fortunately, this heavy squark+gluino background can be suppressed significantly by one of two means, either: (a) we take advantage of the kinematic characteristics inherent in $\tilde{t}^{*} j$, where the extra jet tends to be emitted at large rapidity and high transverse momentum in the detector, and require the hard jet from the squark decay to be similarly far forward; or (b) we veto the extra hard jet from the squark decay, since a sizeable fraction of the signal does not have a high- $p_{T}$ jet. We show the transverse momenta and rapidity distributions of the extra jet in signal $\tilde{t}_{1} \tilde{b}_{1}^{*} j$ production in Fig. 3 for illustration. To be forward-tagged, we require $p_{T}(j)>30 \mathrm{GeV}$ and $3<|\eta(j)|<5$. This provides a suppression factor of 50 , while $1 / 3$ of the signal survives. This would make the background approximately a factor 15 larger than the signal - a mediocre ratio, but not immediately to be dismissed. For a jet veto, which is more speculative and would require far more in-depth investigation of additional QCD radiation in the signal, we veto if $p_{T}(j)>40 \mathrm{GeV}$ and $|\eta(j)|<5$. This veto brings the background down by a factor 113 , while $72 \%$ of the signal survives, for an overall signal-tobackground $(\mathrm{S} / \mathrm{B})$ ratio of about $1 / 3.3$, which is excellent. The $\mathrm{S} / \mathrm{B}$ ratio is superior in the jet veto case because about twice as much signal survives, while the background rejection due to the hard squark decay jet is more than twice as good. Of course, our cuts values for these were not optimized, but serve as a useful rough guide for the moment.

\footnotetext{
${ }^{4}$ Note that $\tilde{q}_{R}$ production is not a background as right-handed squarks cannot decay to charginos.
} 

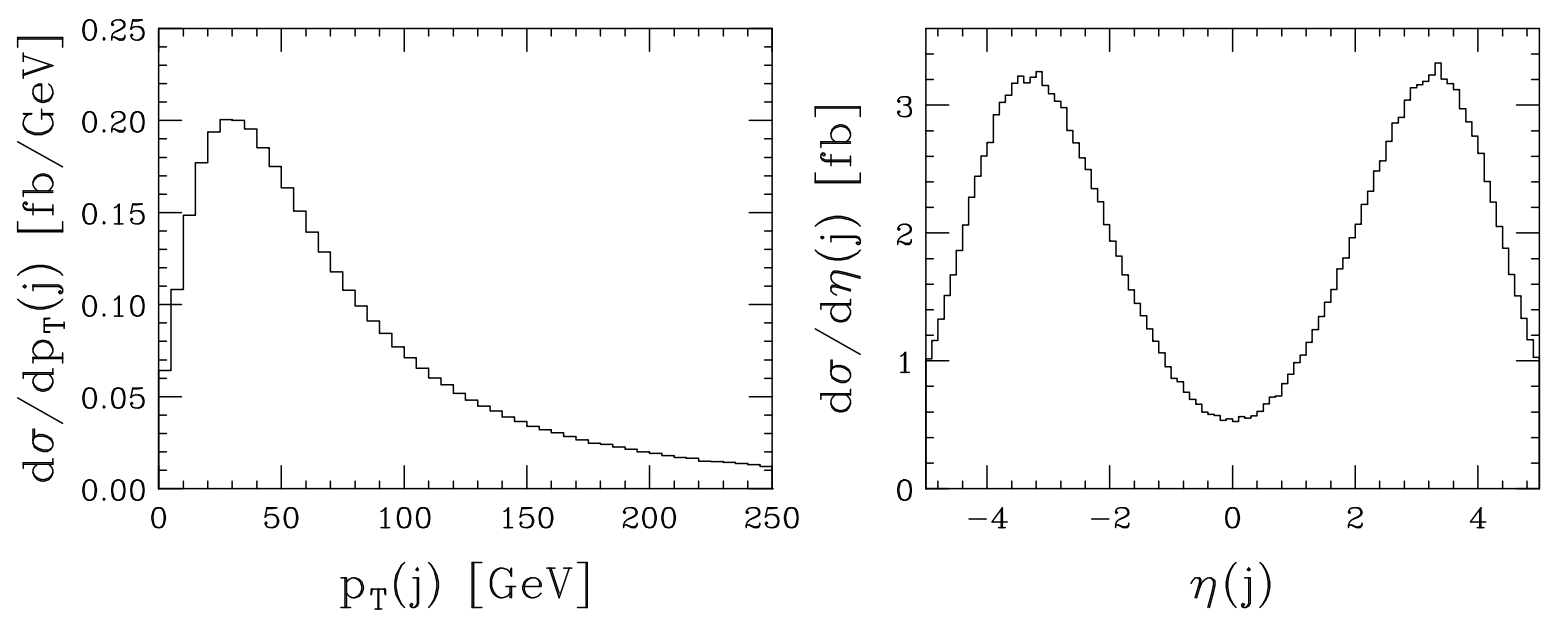

FIG. 3: Transverse momentum and rapidity distributions of the extra jet in $\tilde{t}_{1} \tilde{b}_{1}^{*} j$ production for the SPS5 scenario at the LHC. The ATLAS and CMS calorimeters cannot detect jets beyond a rapidity of 5 , or for $p_{T} \lesssim 20 \mathrm{GeV}$.

We also calculate the rates of $\tilde{b} \tilde{b}^{*} j$ production using SMADGRAPH, and find that there is a factor 7 rejection of this background if one requires a forward jet tag. This would likely be even better, as $\tilde{b} \tilde{b}^{*} j j$ is also quite large. However, the rate for sbottom pair events to produce even one extra jet anywhere in the fiducial volume of the detectors and with $p_{T}(j)>40 \mathrm{GeV}$ is larger than either simple, parton-level $\tilde{b}_{i} \tilde{b}_{i}^{*}$ rate at LO. While there are large uncertainties associated with such QCD calculations at LO, this is not entirely unreasonable, because the NLO corrections to sbottom pair production are quite large [28]. What our result suggests is that the probability to produce an extra hard jet in squark pair events is extremely large, which would only enhance the rejection factor in the jet veto analysis. A similar result holds for squark+gluino production. This issue is sufficiently complicated to be beyond the scope of this paper, therefore is being investigated elsewhere [31].

While we have not attempted to calculate the higher-order QCD $(+1 \mathrm{j})$ contributions to the signal, we anticipate they are much smaller than for the QCD processes, because no color is exchanged between the two incoming partons. Thus, additional radiation patterns should be moderate in comparison to QCD. This will be investigated in future work [32]. For now, our conclusion is that it is appropriate to ignore the sbottom pair backgrounds, as their rejection factors due to both hard QCD radiation and the extra $W$ boson certainly make them very small corrections to the extremely large squark+gluino background, as well as smaller than the signal. We will therefore focus on the squark+gluino backgrounds for our estimates of the feasibility of detecting the signal.

\section{Numerical estimates}

It is beyond the scope of this first-state work to calculate the signal and various backgrounds in full detail, with decays to a detector final state (even at the parton level) and applying kinematic cuts. We relegate this next logical state to future work [32] and here make only estimates of the upper bounds on signal cross sections. To make the estimates reasonable, we base them on branching ratios to the desired final state, as well as the known detector efficiencies for $b$ jet tagging and hadronic tau or lepton ID, depending on the exact final state. 
Our assumptions regarding the SLHC are that it collects $6000 \mathrm{fb}^{-1}$ (two experiments combined) and achieves the particle ID efficiencies discussed previously. We use our LO signal cross sections but NLO squark+gluino background numbers, since the corrections are large; this helps make our estimate conservative. We assume that the $t \bar{t} W$ background is eliminated completely, since it is the smallest to begin with, and ignore the sbottom pair background as discussed above. Thus, the numbers of signal and background events in the forward-tagged jet (jet veto) experiments are roughly 160(320) and 2350(1035). The statistical significance for the forward jet tag case is $\sim 3 \sigma$, while for the jet veto option is $\sim 10 \sigma$. This corresponds to an approximately $12 \%$ uncertainty on the cross section from the veto analysis alone, half of that as the uncertainty on the weak $W-\tilde{t}-\tilde{b}$ coupling. Of course, this assumes that $100 \%$ of the signal would be retained after kinematic cuts, which is obviously not correct. We would nevertheless expect a high retention rate because the finalstate particles originate from the decays of very heavy objects, thus will appear centrally and at high transverse momentum in the detectors with a consequent high probability to pass even conservative kinematic acceptance cut requirements. We would anticipate loss of signal due to cuts to be less than a factor of two. Thus, our estimate is cause for optimism and invites more detailed investigation. One should realize, too, that the signal and background could behave differently with respect to the final-state kinematic cuts, so there is some additional systematic uncertainty at this stage as to the correct $\mathrm{S} / \mathrm{B}$ ratio.

\section{B. SPS1a}

The benchmark point SPS1a has the next-largest stop-sbottom cross section, about half the rate of the SPS5, as shown in Table II. This is primarily because the lightest stop, $\tilde{t}_{1}$, is more massive than in SPS5. The QCD squark and gluino pair background is much larger than in SPS5, in constrast, because the other squarks and gluino are less massive than in SPS5. However, production cross section numbers can be misleading because the different spectrum results in different BRs of the various states. In addition, the stop mixing is somewhat different, so that the $\tilde{t}_{2}$ cross sections are comparable to $\tilde{t}_{1}$, as we saw in Table 【. Here, $\tilde{t}_{1} \rightarrow b \chi_{1}^{+}$only $2 / 3$ of the time, compared to always in SPS5, while $\tilde{b}_{1} \rightarrow \bar{t} \chi_{1}^{+}$occurs at three times the BR of SPS5. In SPS1a, there are also non-trivial contributions to the same final state from the sizeable $\tilde{t}_{2}$ rate. In the backgrounds, while there is far larger squarkgluino rate than in SPS5, the gluino BRs to bottom-sbottom and top-stop are a factor of several smaller. As with SPS5, for SPS1a we summarize in Table IV the sparticle masses, total widths at NLO in QCD, and major branching fractions relevant for this analysis.

We proceed as we did for the SPS5 scenario previously, constructing two possible analyses, one demanding the presence of a far-foward tagging jet, the other vetoing any additional hard jets, which the heavy squark decays would give. The rejection factors against the squark-gluino backgrounds are 40 and 80, respectively, which are about 4/5 of the factors in SPS5. Signal retention rates for both possible analyses are similar to those in SPS5.

At this point, we estimate after all BRs, detector ID efficiencies, and including the NLO corrections for the heavy squark and gluino backgrounds, that the forward jet tag analysis could gather up to about 90 events in $6000 \mathrm{fb}^{-1}$, against a background of about 3200 events, while the jet veto analysis could retain up to about 220 signal events against a background of 1600. Kinematic cuts on the final state particles will reduce this somewhat, but again we argue retention is likely to be highly efficient as the identifiable particles come from the decays of very heavy objects and thus tend to be highly boosted. While the tagged analysis 


\begin{tabular}{|c|c|c|c|}
\hline & $m$ & $\Gamma_{\mathrm{NLO}}$ & $\mathrm{BR}\left(q \chi_{1}^{+}\right)$ \\
\hline$\tilde{t}_{1}$ & 396 & 1.92 & $68.1 \%$ \\
$\tilde{t}_{2}$ & 587 & 7.06 & $23.6 \%$ \\
$\tilde{b}_{1}^{*}$ & 517 & 3.77 & $44.5 \%$ \\
$\tilde{b}_{2}^{*}$ & 547 & 0.875 & $20.6 \%$ \\
$\tilde{u}_{L}$ & 568 & 5.53 & $65.0 \%$ \\
$\tilde{d}_{L}^{*}$ & 573 & 5.34 & $60.8 \%$ \\
\hline
\end{tabular}

\begin{tabular}{|c|c|c|c|c|}
\hline & $m$ & $\Gamma_{\mathrm{NLO}}$ & $\mathrm{BR}\left(b b_{1}^{*}\right)$ & $\mathrm{BR}\left(\bar{t} \tilde{t}_{1}\right)$ \\
\hline$\tilde{g}$ & 607 & 4.33 & $10.4 \%$ & $5.40 \%$ \\
\hline \hline & $m$ & $\Gamma_{\mathrm{LO}}$ & $\mathrm{BR}\left(\nu_{\tau} \tilde{\tau}^{+}\right)$ & $\mathrm{BR}\left(W^{+} \chi_{1}^{0}\right)$ \\
\hline$\chi_{1}^{+}$ & 181 & 0.0154 & $95.3 \%$ & $4.7 \%$ \\
\hline \hline & $m$ & $\Gamma_{\mathrm{LO}}$ & $\mathrm{BR}\left(\tau \chi_{1}^{0}\right)$ & \\
\hline$\tilde{\tau}_{1}^{+}$ & 136 & 0.155 & $100 \%$ & \\
\hline
\end{tabular}

TABLE IV: MSSM particle masses and widths [GeV], and important branching ratios for scenario SPS1a. The LSP is the lightest neutralino, $\chi_{1}^{0}$, with mass $97.4 \mathrm{GeV}$.

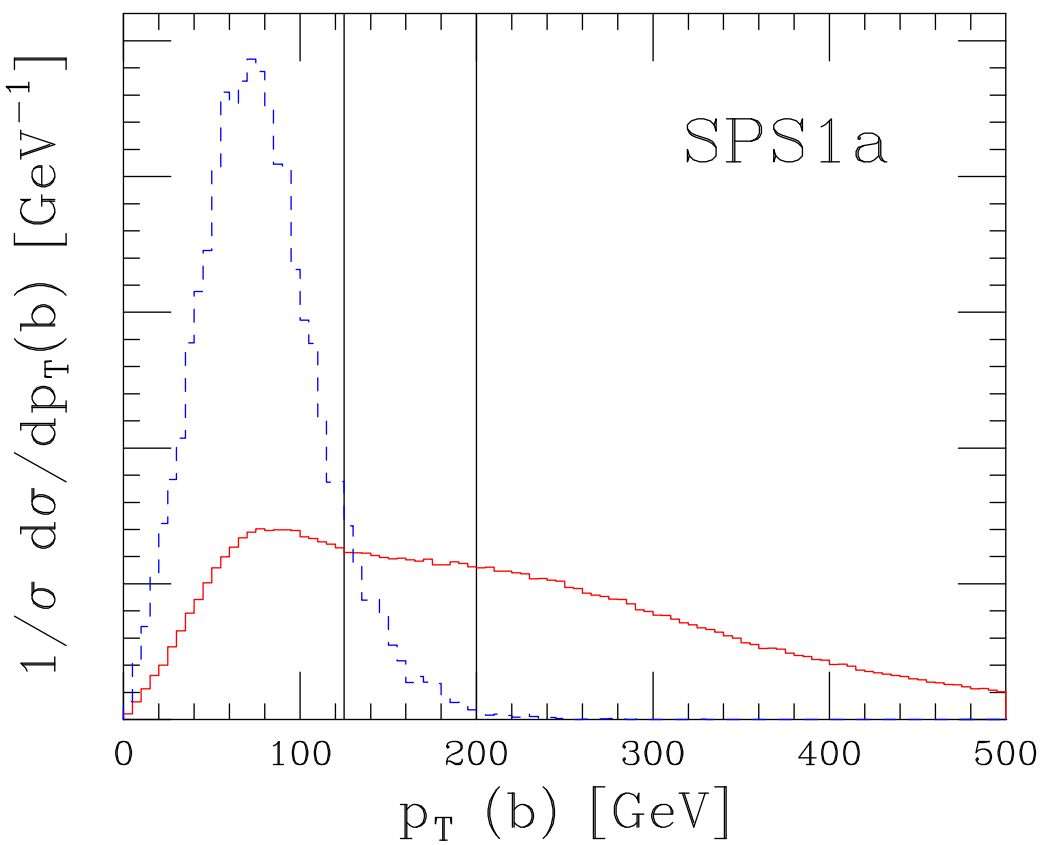

FIG. 4: Transverse momentum $\left(p_{T}\right)$ distribution of the $b$ jet which does not come from a top quark decay, in the SPS1a scenario at the LHC. Signal $\tilde{t_{1}} \tilde{b}_{1}^{*}$ events are shown by the solid red curve and $\tilde{u}_{L} \tilde{g} \rightarrow \chi_{1}^{+} b \tilde{b}_{1}^{*}$ background events by the dashed blue curve. The vertical lines are two possible kinematic cuts: the left-hand cut would reduce the background by a factor 10 , with a $29 \%$ loss of signal, while the right-hand cut would yield a factor 270 background reduction, at a cost of half the signal.

is quite poor, the veto analysis already could achieve up to $\sim 5 \sigma$, with up to a statistical uncertainty on the signal cross section of about $20 \%$.

One can improve on this by carefully considering the kinematic characteristics of both signal and background. The squark+gluino rate can be broken down into two major parts: $\tilde{g} \rightarrow b \tilde{b}^{*}$, and $\tilde{g} \rightarrow \tilde{t} \tilde{t}$; they are roughly equal. The bottom quark jet in gluino decays, however, is extremely soft, due to the small mass splitting between the gluino and the sbottom. The $b$ jet arising from stop decays in the signal, however, is quite hard, due to the rather large mass splitting between stop and chargino.

For this first-stage study we implement one level of decays with SMADGRAPH and calculate the rates for the signal process $\tilde{t} \tilde{b}^{*} \rightarrow b \chi_{1}^{+} \bar{t} \chi_{1}^{+}$as well as the background processes $\tilde{u}_{L} \tilde{g} \rightarrow$ $d \chi_{1}^{+} b \tilde{b}_{1}^{*}, d \chi_{1}^{+} \tilde{t}_{1}$. We use the narrow width approximation for phase space, as the sparticle 


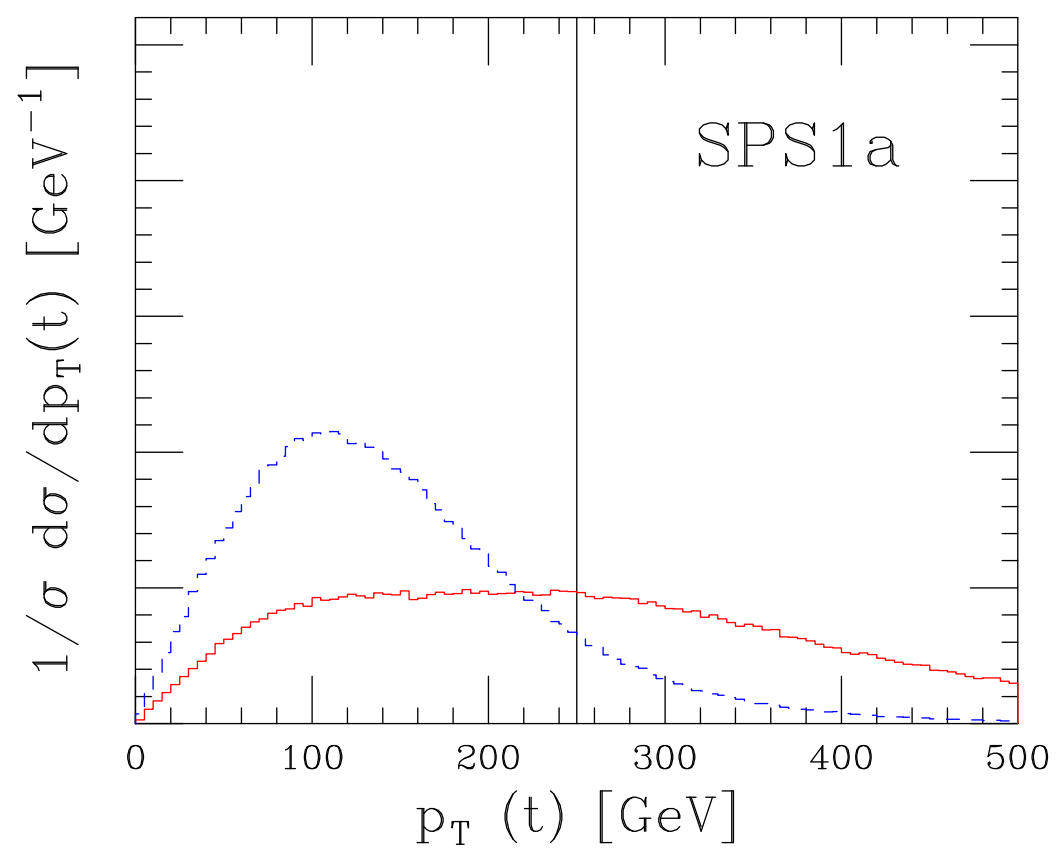

FIG. 5: Transverse momentum $\left(p_{T}\right)$ distribution of the top quark in the SPS1a scenario at the LHC. Signal $\tilde{t_{1}} \tilde{b}_{1}^{*}$ events are shown by the solid red curve and $\tilde{u}_{L} \tilde{g} \rightarrow \chi_{1}^{+} \tilde{t}_{1}$ background events by the dashed blue curve. The vertical line represents a possible kinematic cut which would eliminate $88 \%$ of the background at a cost of half the signal.

widths are typically a couple percent of their mass (see TableIV), but implement full matrix elements to retain spin angular correlations in the backgrounds, using total widths for the various particles as calculated at LO (to match the LO couplings in SMADGRAPH) by the program SDECAY 29]; we do consider overall BRs at NLO, also given by SDECAY, since the corrections for the gluino can be large [30]. We show the $p_{T}$ distribution of the $b$ jet not arising from the top quark decay in Fig. 4. There is a dramatic difference in the kinematic behavior, as expected ${ }^{5}$. We display two possible cuts, one which reduces the background by a factor 10 while retaining $71 \%$ of the signal, the other which totally obliterates the background (factor 270) at the expense of fully half the signal. Note that imposing such a cut would also reduce the $\tilde{g} \rightarrow \bar{t} \tilde{t}$ rate by the approximately the same amount as the signal, as the $b$ jet not from top quark decay there comes from a stop as in the signal, so will display a similar kinematic behavior. A nice feature of the signal distribution is its relative flatness to high $p_{T}$ : if the tail of the background distribution is wider than expected, one can adjust the cut to compensate for this at very little relative cost to the signal.

Analogously for the other background contribution, $\tilde{g} \rightarrow \tilde{t} \tilde{t}$, the top quark daughter will tend to be boosted more in the signal, where it was produced in conjuction with a fairly light chargino, whereas in the background its decay partner is a relatively heavy stop. Although the differences are not as dramatic as with the $b$ jet in stop v. gluino decays, we see in Fig. [5 that it is possible to find a cut which reduces the background by about an order of magnitude while retaining $50 \%$ of the signal. This is a somewhat higher price to pay than in the gluino decay to bottom-sbottom, but it does improve the overall statistical significance,

\footnotetext{
${ }^{5}$ We examined this also for SPS5, but these kinematic features were not as dramatically different there.
} 
as well as the more important signal-to-background ratio. Again, imposing this cut would reduce the $\tilde{g} \rightarrow b \tilde{b}^{*}$ portion of the background similar to the signal.

Taking both cuts together, we estimate up to about 32(78) signal events could be retained in the forward-tag (jet veto) analysis, with a background of about 210(105) events. (Note that this is rather pessimistic, since the two cuts are not truly orthogonal, thus the actual signal retention rate should be higher.) This improves the jet veto case to a very respectable $\mathrm{S} / \mathrm{B}$ ratio of $1 / 1.3$, up to about $7.6 \sigma$ statistical significance, and potentially up to a $20 \%$ statistical uncertainty on the signal cross section. This is again idealized, but we would expect a high signal retention rate after cuts, as most of the final-state particles will be highly boosted and register well in the detectors. Again, as with SPS5 there is some systematic uncertainty on S/B that arises from not knowing whether the signal and background behave similarly for the final-state kinematic cuts. Further study will clarify this.

\section{General MSSM scenarios}

While the SPS scenarios constitue a useful framework in which to study SUSY phenomenology at upcoming collider experiments, we remind ourselves that they do not by any means fully represent the plethora of possible MSSM parameterizations. They are only starting points: the SUSY breaking schemes mSUGRA, gauge-mediated (GMSB) and anomaly-mediated (AMSB) all make broad assumptions about unification of input mass parameters at some high scale. While the motivations for doing so are elegant, they are not by any stretch of the imagination certain.

Based on our experiences studying SPS5 and SPS1a, we can draw a few simple conclusions to guide exploration of parameter space with a more open mind. First, and most obviously, LHC has the potential to observe stop-sbottom production when the cross sections are large enough to produce a statistically useful number of events; the backgrounds in the SPS cases we examined so far can be suppressed to approximately the level of the signal. For the production of heavy objects, LHC is limited mostly by phase space. Thus, scenarios with lighter stops and sbottoms are more desireable.

Second, very large backgrounds arise from first- and second-generation squark+gluino and gluino pair production, but they can also be heavily suppressed using additional kinematic information in the events. Scenarios with very heavy squarks and gluinos relative to stops and sbottoms are in some sense variants of SPS5 and highly likely to be accessible by comparison. Alternatively, if the gluino is lighter and cannot decay to stops or sbottoms, then this background completely disappears. The other squarks cannot become a sizeable background on their own, as their decays would not yield heavy flavor quarks (including a top quark) in the final state.

One does have to worry about gluinos lighter than the sbottom, however, as the strong decay $\tilde{b} \rightarrow b \tilde{g}$ then becomes possible, which tends to dominate in BR over the weak decays to bottom quark plus chargino. Thus, while there is no background, there is little signal rate left to the same-sign charged lepton pair we advocate. Were $b$ jet-charge tagging to be possible with decent efficiency, then such a scenario might be accessible. One could also consider the possibility of identifying mixed-flavor production in this scenario by observing a single lepton from the stop decay, two $b$ jet tags, and a characteristic gluino, thus separating the signal from stop pair or sbottom pair production; but this is rather speculative and would require a completely different analysis.

In searching for other viable scenarios, we do not rigorously check for known constraints 
from present data, except for the Higgs and chargino limits from LEP [33, 34, 35, 36], and of course the requirement of a neutral, colorless LSP. Nor do we attempt a thorough, systematic exploration of parameter space at this early stage. Our goal is simply to emphasize how different MSSM realizations could change expectations for the stop-sbottom signal qualitatively. An early warning: scenarios with a small NLSP-LSP mass difference can give final-state taus or leptons which are soft a large fraction of the time, presenting a rate problem after detector acceptance cuts. The exception to this is when the mass difference is so small that the NLSP is long-lived, as we will see below.

- Lighter stop and sbottom via increased mixing. Starting with SPS1a and simply increasing the value of $A_{0}$ to $-500 \mathrm{GeV}$, thus maintaining the unification of input mass parameters, results in about a $70 \%$ cross section increase for the slightly lighter stops and sbottoms. However, the gluino mass doesn't change, while its BR to top-stop increases by a factor three. Signal significane is likely to decrease slightly.

- Heavier gluino via increased $M_{3}$. Starting with the low-energy effective SPS1a point but instead allowing non-universal values for $m_{1 / 2}$, setting $M_{1,2,3}=100,200,500 \mathrm{GeV}$ and $A_{t, b}=-2 \mathrm{TeV}$, we obtain a very heavy gluino, $m_{\tilde{g}}=1154 \mathrm{GeV}$, while the stop and sbottom masses similar to those of SPS1a, and their total production cross sections about $1 / 3$ the SPS1a rate. Now the squark+gluino backgrounds are only a few hundred fb to start, almost two orders of magnitude smaller, but with BRs to stops and sbottoms a factor of a few larger than SPS1a. With this spectrum the kinematic tricks we identified for SPS1a will not work as well, as the gluinosbottom mass splitting is now several hundred GeV. However, the overall lowering of background from production rate, less the increased BRs, is about the same as the suppressed rate of SPS1a via kinematic differences. Such a scenario looks promising only if the gluino BR to stop or sbottom and loss of kinematic cut effectiveness does not grow at a faster rate than the cross section falls off due to phase space.

- A variant of the above with $M_{1,2,3}=150,300,1000 \mathrm{GeV}$ and universal $m_{0}=500 \mathrm{GeV}$ at the $\mathrm{TeV}$ scale predicts a $970 \mathrm{GeV}$ gluino, a light stop of $322 \mathrm{GeV}$ and lightest sbottom of $475 \mathrm{GeV}$. The signal rates are similar to SPS5, with a smaller background than in SPS5, from both smaller production rates and smaller gluino decay rates to stops and sbottoms. We find a wide parameter space around this scenario which produces a similar spectrum.

- Gluino lighter than the sbottoms. Starting at SPS1a but allowing non-universal masses, we lower $m_{1 / 2}$ for $S U(3)$ only to $100 \mathrm{GeV}$, set $A_{0}=0$ and $m_{\tilde{t}_{R}}=m_{\tilde{b}_{R}}=$ $150 \mathrm{GeV}$ at the GUT scale. The gluino mass is now $271 \mathrm{GeV}$, while the lighter sbottom mass is $274 \mathrm{GeV}$. The lightest stop is $193 \mathrm{GeV}$; the heavier stop and sbottom are both under $400 \mathrm{GeV}$. The total of stop-sbottom cross sections is $160 \mathrm{fb}$, with no background from squark+gluino pairs. However, the lighter sbottom does not decay at all to chargino, rather mostly to $b+\mathrm{LSP}$. The $\tilde{b}_{2}$ actually dominates the production rate, and it has a $6 \% \mathrm{BR}$ to chargino. The rate to $b \bar{t} \chi_{1}^{+} \chi_{1}^{+}$is larger than at SPS1a by a small factor. The chargino is lighter than the stau in this scenario, which would decay like a $W$ boson, with an effective overall ID efficiency via its decays to $e, \mu$ about that same as a stau pair. Such scenarios look extremely promising. Other variations on this theme, with $m_{\tilde{g}} \sim m_{\tilde{b}}$, are easy to find. 
Our qualitative survey suggests that parameterizations which give larger signal cross sections are often good, but also sometimes less viable, for a variety of reasons. Usually the culprit is either a dramatically larger BR of the gluino to a stop or sbottom, or the stop and sbottom BRs themselves squeeze the distinctive signal we propose. Analogously, smaller cross sections can sometimes be deceptively good, often for similar reasons.

\section{Stau coannihilation scenario}

We examine a stau-coannihilation scenario [37, 38], inspired by the dark matter relic density 39]. We choose $\mathrm{TeV}$-scale input parameters of $M_{1,2,3}=100,100,800 \mathrm{GeV}, m_{0}=$ $800 \mathrm{GeV}$ for the first two generations, $m_{\tilde{\tau}}=127 \mathrm{GeV}, m_{Q_{L}}=m_{t_{R}}=400 \mathrm{GeV}, m_{b_{R}}=$ $300 \mathrm{GeV}$ and $A_{t, b}=-700 \mathrm{GeV}$. The stau mass obtained is $100 \mathrm{MeV}$ above the LSP at $97 \mathrm{GeV}$, with a $101 \mathrm{GeV}$ chargino - this is the principal features of this scenario. As a consequence, the stau is relatively long-lived. We selected other parameters to guarantee reasonably light third-generation squarks: the lighter stop and sbottom are 243 and $288 \mathrm{GeV}$, respectively, with their heavier partners at 544 and $406 \mathrm{GeV}$. The other squarks and gluino are somewhat heavier at $810 \mathrm{GeV}$. With regard to the colored sparticle sector, this point is not all that different from SPS5.

The largest signal cross section is $\tilde{t}_{1} \tilde{b}_{2}^{*}$ at $67 \mathrm{fb}$, as shown in Table $\nabla$. The stop decays $100 \%$ to our desired chargino, and the sbottom $1 / 4$ of the time. The chargino in turn decays exclusively to the lighter stau, which is long-lived. Herein lies the munificence: the detection efficiency for each stau will be extremely close to $100 \%$, with perfect charge identification. Already, then, any such scenario will achieve at least a factor 4 detection efficiency improvement over any model where the stau decays promptly to a tau. Moreover, there is no SM background at all. For a jet tag strategy, we calculate $2.63 \mathrm{fb}$ for the signal final state $b \bar{t} \tilde{\tau}_{1}^{+} \tilde{\tau}_{1}^{+}$where we include a factor $2 / 3$ for the hadronic BR of the top quark. For a jet veto strategy, we calculate $4.13 \mathrm{fb}$.

Squark+gluino backgrounds are similar to SPS5, but with $50 \%$ BR of gluino to bottomsbottom and top-stop of the $(++)$ charge sign, larger than SPS5. As implied, the gluino does not decay to any first- or second-generation squark plus quark, thus removing gluino pair production as a background. Rejection factors for the jet tag and jet veto scenarios are extremely good: 60 and 200, respectively. By the time NLO corrections, jet tag/veto rejection factors and BRs are taken into account, we find about $2.09 \mathrm{fb}$ for the jet tag case and $0.61 \mathrm{fb}$ using instead a jet veto. These cross sections are smaller than the signal.

However, the real background comes from QCD sbottom pair production. Specifically, $\tilde{b}_{2} \tilde{b}_{2}^{*}$ with a NLO cross section of approximately $1.9 \mathrm{pb}$, as $\tilde{b}_{2} \tilde{b}_{2}^{*}$ cannot fake the signal at this point. After BRs, this is about $170 \mathrm{fb}$, although this is comparable to the sbottom pair rate for SPS5. The difference here is that the squark+gluino background is smaller, rather than significantly larger. Again we assume that there is an order of magnitude rejection of sbottom pairs via the extra $W$ boson, and another factor of 5 from a jet veto on the extra radiation. There is a large uncertainty associated with this, but as we will see, it is largely irrelevant for observational success at this point.

Because of the anticipated large detection efficiency for long-lived staus, about a factor 5 over staus which would promptly decay to taus, our rough estimate of observability here will be for the LHC already, rather than the SLHC. Using a jet veto strategy we would anticipate approximately 620 double-b-tagged signal events (including hadronic top decay), on top of a total background of about 400 events; S/B would be better than 1/1. This would provide for a remarkable $>30 \sigma$ detection and statistical uncertainty on the signal cross section to this final state of about 5\%. Even if the background rejection factors due to $W$ or jet veto 


\begin{tabular}{|c|c|c|c|c|}
\hline & $\tilde{t}_{1} \tilde{b}_{1}$ & $\tilde{t}_{1} \tilde{b}_{2}$ & $\tilde{t}_{2} \tilde{b}_{1}$ & $\tilde{t}_{2} \hat{b}_{2}$ \\
\hline$s$-ch., $\tilde{t} \tilde{b}^{*}$ & 0.69 & 9.80 & 0.11 & 2.19 \\
\hline$s$-ch., $\tilde{t}^{*} b$ & 0.32 & 4.33 & 0.05 & 0.84 \\
\hline$t$-ch., $\tilde{t} \tilde{b}^{*}$ & 2.28 & 57.2 & 0.95 & 8.18 \\
\hline$t$-ch., $\tilde{t}^{*} \tilde{b}$ & 1.21 & 28.6 & 0.44 & 3.74 \\
\hline
\end{tabular}

TABLE V: Cross sections [fb] for mixed stop-sbottom production at LHC in a stau-coannihilation scenario. For the $t$-channel results, we do not impose a kinematic cut on the final-state quark.

\begin{tabular}{|c|c|c|c|}
\hline & $m$ & $\Gamma_{\mathrm{NLO}}$ & $\mathrm{BR}\left(q \chi_{1}^{+}\right)$ \\
\hline$\tilde{t}_{1}$ & 243 & 0.87 & $100 \%$ \\
$\tilde{t}_{2}$ & 544 & 10.7 & $26.0 \%$ \\
$\tilde{b}_{1}^{*}$ & 288 & 0.19 & $6.02 \%$ \\
$\tilde{b}_{2}^{*}$ & 406 & 6.30 & $25.8 \%$ \\
$\tilde{u}_{L}$ & 806 & 9.3 & $66.0 \%$ \\
$\tilde{d}_{L}^{*}$ & 810 & 9.2 & $64.1 \%$ \\
\hline
\end{tabular}

\begin{tabular}{|c|c|c|c|c|}
\hline & $m$ & $\Gamma_{\mathrm{NLO}}$ & $\mathrm{BR}\left(b b^{*}\right)$ & $\mathrm{BR}(\tilde{t} \tilde{t})$ \\
\hline$\tilde{g}$ & 810 & 31.4 & $30 \%$ & $20 \%$ \\
\hline \hline & $m$ & $\Gamma_{\mathrm{LO}}$ & $\mathrm{BR}\left(\nu_{\tau} \tilde{\tau}^{+}\right)$ & \\
\hline$\chi_{1}^{+}$ & 101 & 0.00115 & $100.0 \%$ & \\
\hline
\end{tabular}

TABLE VI: MSSM particle masses and widths [GeV], and important branching ratios for a dark matter-motivated stau coannihilation scenario with squark and gluino masses similar to SPS5, but with a slightly lighter $\tilde{b}_{2}$. The LSP is the lightest neutralino, $\chi_{1}^{0}$, with mass $97.0 \mathrm{GeV}$. The stau is the NLSP, with mass $97.1 \mathrm{GeV}$, and is long-lived on detector timescales..

turn out to be off by an order of magnitude, this would still allow for a $10 \sigma$ observation and up to an $11 \%$ statistical uncertainty on the cross section.

Needless to say, at SLHC this channel would rapidly become dominated by systematic errors, such as the QCD uncertainty on the signal theoretical cross section or the measurement of the $\tilde{b}_{2} \tilde{b}_{2}^{*}$ rate and its BRs. However, because the dominant background is likely this squark pair rather than squark+gluino production, there is an additional handle one could apply to improve the situation: the signal has an approximately $2: 1$ asymmetry for $(++) \mathrm{v}$. (- -) production, due to the predominance of initial-state valence $u$ over valence $d$ partons. QCD sbottom pair production is totally symmetric, in contrast. Thus, by measuring the charge asymmetry of the final state,

$$
A=\frac{\sigma_{++}-\sigma_{--}}{\sigma_{++}+\sigma_{--}}
$$

one could gain additional strong leverage over residual systematic uncertainty of the QCD background.

\section{GMSB scenario}

A particularly interesting scenario is gauge-mediated SUSY breaking (GMSB), which has a gravitino LSP. Because the decay of the NLSP would be of gravitational strength, the NLSP is typically long-lived, and if charged would be extraordinarily noticeable in experiment as a heavy charged object passing through the muon chambers. Because of this, the efficiency to detect SUSY events would be very close to $100 \%$ if the NLSP is charged, completely eliminating SM backgrounds and retaining nearly the whole rate of stop-sbottom pairs, modulo the efficiency for $b$-tagging, needed to identify that the SUSY signal comes from third-generation squarks. 
SPS7 is a GMSB scenario with stau NLSP, but because of the very large stop and sbottom masses, there is practically no rate for mixed-flavor pairs at LHC. We instead look for parameterizations of GMSB where the stops and sbottoms are light enough to be produced at the LHC, and also where the gluino is slightly lighter than the sbottoms, so that the $\operatorname{BR}(\tilde{g} \rightarrow b \tilde{b})$ does not dominate. SUSY backgrounds are then composed only of light squarks and gluinos which decay to same-sign staus, with light jets mistagged as $b$ jets. The large suppression of such fakes may make such a scenario feasible for much smaller signal cross sections than in SPS1a or SPS5.

We easily find an example of such a parameterization, by choosing $M_{\text {mes }}=100 \mathrm{TeV}$, $M_{S U S Y}=20 \mathrm{TeV}, \tan \beta=5, \mu<0$, and the presence of 3 lepton and 2 quark messenger states. The latter is a somewhat odd choice, but is not restricted in any way. For these choices, $m_{\tilde{t}_{1}, \tilde{t}_{2}, \tilde{b}_{1}, \tilde{b}_{2}}=350,410,360,370 \mathrm{GeV}$. Only the $\tilde{b}_{2}$ cross sections are of decent size:

that for $\tilde{t}_{1} \tilde{b}_{2}^{*}$ is about $1 \mathrm{fb}$ and that for $\tilde{t}_{2} \tilde{b}_{2}^{*}$ is about $3.6 \mathrm{fb}$, both obtained by requiring the forward-tagged jet as previously discussed. This would produce $\mathcal{O}(50)$ events at SLHC, including detector efficiencies but no kinematic cuts, which should be highly efficient given that the $b$ quarks would come from the decays of very heavy states.

\section{$\underline{\text { AMSB scenario }}$}

Another interesting SUSY scenario is that of anomaly-mediation (AMSB), which typically possesses the characteristic of very small mass splitting between the LSP and NLSP, which are usually the lightest neutralino and chargino, respectively. The NLSP can exhibit dramatic vertex displacements in the detector of many centimeters before decaying. Because of this, SM backgrounds would be non-existent and the efficiency for capturing AMSB events would be extremely high, close to $100 \%$, as in some GMSB scenarios. Charginos would decay to $e$ and $\mu$ each about $1 / 7$ of the time, giving an overall clean, charge-identifiable state about $8 \%$ of the time in stop-sbottom events.

We do not explore AMSB parameter space widely, but find in general that it is difficult to obtain light-enough squark masses for LHC stop-sbottom production to be large enough to be useful, while at the same time avoiding the LEP constraints on the chargino mass.

\section{OUTLOOK}

Our conclusions from this initial study relevant for LHC/SLHC are threefold. First, it does appear that there is a path to separately measuring weak production of mixed-flavor third-generation squark pairs in many SUSY scenarios at the LHC/SLHC. If further, more detailed studies bear this out, it represents a significant increase in the physics capability of LHC. Second, the interpretation of weak cross section measurements would depend strongly on what additional information is extractable from other production processes. The signals proposed here could represent a way to help disentangle third-generation squark mixing, at worst, or a potential measurement of the weak vertex and the flavor matrix that diagonalizes squarks, relative to the CKM matrix of the Standard Model. This would depend mostly what variety of SUSY is realized in nature, if at all: the relative spectrum, the overall scale of SUSY masses, and other unforeseeables. While hints of generational squark mixing may show up in QCD production channels, measuring the weak production vertex would greatly aid in testing the flavor-diagonal hypothesis and measuring deviations from it. Third, any scenario which has a long-lived NLSP is a boon for this measurement, as it would improve upon the detection efficiency of the signal by (at a minimum) a factor 4-5. Thus, any given 
model with squark and gluino masses that would appear to have a viable measurement of stop-sbottom production at SLHC would probably become viable at LHC if it also had a long-lived NSLP.

Finally, we observe that our ability to pursue this interesting physics is limited only by cross section. Because squarks are typically heavy, copious production at future colliders could potentially open a new window onto these deeper questions, if SUSY is found in nature. One path is a next-generation (or beyond) linear collider [9, 40], which could study the decays of squark pairs with great precision to help disentangle the mixing angles [41]. Another possible path would be a higher-energy hadron collider such as the proposed VLHC [42], which would have the ability to produce mixed-flavor squark pairs with cross sections typically two order of magnitude larger than at LHC.

\section{Acknowledgements}

We thank Tilman Plehn and Matt Strassler for highly useful discussions and critiques, Howie Baer for suggestions about additional SUSY backgrounds, and to Sally Dawson, Peter Zerwas and Lynne Orr for reviews of the manuscript. This research was supported in part by the U.S. Department of Energy under grant No. DE-FG02-91ER40685.

\section{Note added in proof:}

After submission of this work another paper appeared on the arXiv which also calculates the $s$-channel stop-sbottom cross sections [43]. Their calculations for this channel agree with ours.

[1] For reviews of supersymmetry, see: S. P. Martin, arXiv:hep-ph/9709356,

I. J. R. Aitchison, arXiv:hep-ph/0505105.

[2] R. Haag, J. T. Lopuszanski and M. Sohnius, Nucl. Phys. B 88, 257 (1975).

[3] P. Bechtle, K. Desch and P. Wienemann, arXiv:hep-ph/0412012,

[4] R. Lafaye, T. Plehn and D. Zerwas, arXiv:hep-ph/0404282.

[5] B. K. Gjelsten, D. J. Miller and P. Osland, arXiv:hep-ph/0501033

[6] I. Hinchliffe, F. E. Paige, M. D. Shapiro, J. Soderqvist and W. Yao, Phys. Rev. D 55, 5520 (1997).

[7] H. Bachacou, I. Hinchliffe and F. E. Paige, Phys. Rev. D 62, 015009 (2000).

[8] B. C. Allanach, C. G. Lester, M. A. Parker and B. R. Webber, JHEP 0009, 004 (2000).

[9] T. Abe et al. [American Linear Collider Working Group], in Proc. of the APS/DPF/DPB Summer Study on the Future of Particle Physics (Snowmass 2001) ed. N. Graf, arXiv:hep-ex/0106056 J. A. Aguilar-Saavedra et al. [ECFA/DESY LC Physics Working Group], arXiv:hep-ph/0106315 K. Abe et al. [ACFA Linear Collider Working Group], arXiv:hep-ph/0109166.

[10] G. Weiglein et al. [LHC/LC Study Group], arXiv:hep-ph/0410364

[11] F. Gianotti et al., Eur. Phys. J. C 39, 293 (2005). 
[12] For a review of top quark physics, see: D. Chakraborty, J. Konigsberg and D. L. Rainwater, Ann. Rev. Nucl. Part. Sci. 53, 301 (2003).

[13] ATLAS TDR, report CERN/LHCC/99-15 (1999).

[14] CMS TP, report CERN/LHCC/94-38 (1994).

[15] R. M. Barnett, J. F. Gunion and H. E. Haber, Phys. Rev. D 37, 1892 (1988); UCD-88-30, Snowmass: DPF Summer Study 1988:0230; Phys. Lett. B 315, 349 (1993).

[16] M. J. Duncan, Nucl. Phys. B 221, 285 (1983).

[17] J. F. Donoghue, H. P. Nilles and D. Wyler, Phys. Lett. B 128, 55 (1983).

[18] T. Stelzer and W. F. Long, Comput. Phys. Commun. 81, 357 (1994).

[19] K. Hagiwara, J. Kanzaki, T. Plehn, D. Rainwater and T. Stelzer, in preparation.

[20] J. Pumplin et al., JHEP 0207, 012 (2002)

[21] B. C. Allanach et al., in Proc. of the APS/DPF/DPB Summer Study on the Future of Particle Physics (Snowmass 2001) ed. N. Graf, Eur. Phys. J. C 25, 113 (2002).

[22] For a review of SUSY model building, see: M. Drees and S. P. Martin, arXiv:hep-ph/9504324.

[23] S. Dawson, E. Eichten and C. Quigg, Phys. Rev. D 31, 1581 (1985).

[24] D. L. Rainwater, D. Zeppenfeld and K. Hagiwara, Phys. Rev. D 59, 014037 (1999).

[25] F. Maltoni, D. L. Rainwater and S. Willenbrock, Phys. Rev. D 66, 034022 (2002).

[26] U. Baur, A. Juste, L. Orr and D. Rainwater, in preparation.

[27] W. Beenakker, R. Hopker, M. Spira and P. M. Zerwas, Nucl. Phys. B 492, 51 (1997).

[28] W. Beenakker, M. Kramer, T. Plehn, M. Spira and P. M. Zerwas, Nucl. Phys. B 515, 3 (1998).

[29] M. Muhlleitner, A. Djouadi and Y. Mambrini, arXiv:hep-ph/0311167.

[30] W. Beenakker, R. Hopker, T. Plehn and P. M. Zerwas, Z. Phys. C 75, 349 (1997).

[31] T. Plehn, D. Rainwater and P. Skands, in preparation.

[32] D. Berdine and D. Rainwater, in preparation.

[33] R. Barate et al. [ALEPH Collaboration], Phys. Lett. B 565, 61 (2003).

[34] LEP Higgs Working Group Note 2004-01, Aug. 2004; there is as yet no final combinedexperiments, published result.

[35] B. Clerbaux, Nucl. Phys. Proc. Suppl. 115, 378 (2003).

[36] G. Degrassi, S. Heinemeyer, W. Hollik, P. Slavich and G. Weiglein, Eur. Phys. J. C 28, 133 (2003).

[37] J. R. Ellis, T. Falk and K. A. Olive, Phys. Lett. B 444, 367 (1998).

[38] J. R. Ellis, T. Falk, K. A. Olive and M. Srednicki, Astropart. Phys. 13, 181 (2000) [Erratumibid. 15, 413 (2001)].

[39] G. Bertone, D. Hooper and J. Silk, Phys. Rept. 405, 279 (2005); and references therein.

[40] C. P. W. Group et al., arXiv:hep-ph/0412251.

[41] A. Bartl et al., Z. Phys. C 76, 549 (1997).

[42] U. Baur et al., in Proc. of the APS/DPF/DPB Summer Study on the Future of Particle Physics (Snowmass 2001) ed. N. Graf, eConf C010630, E4001 (2001) arXiv:hep-ph/0201227.

[43] G. Bozzi, B. Fuks and M. Klasen, Phys. Rev. D 72, 035016 (2005). 


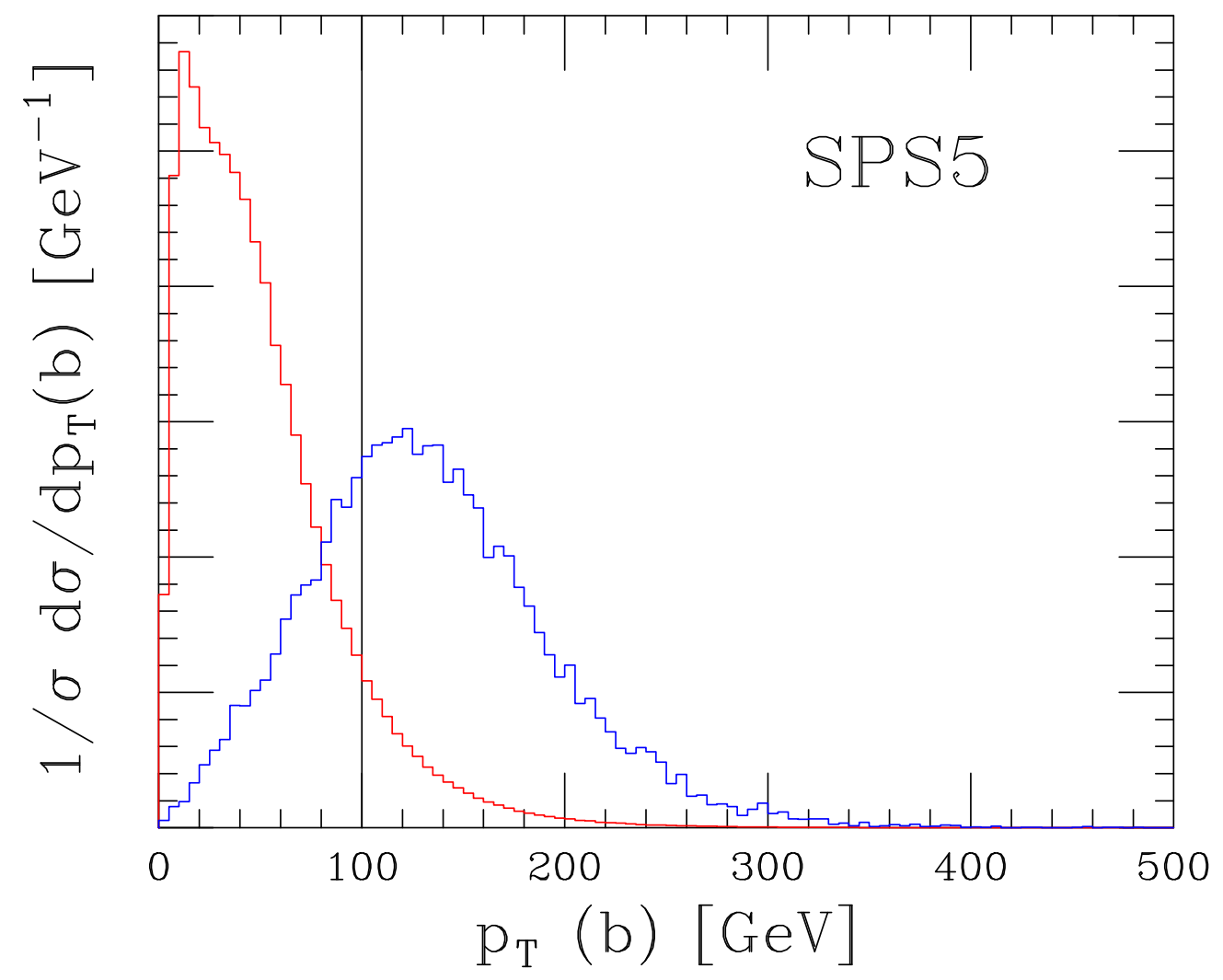

